\title{
Basal abelisaurid and carcharodontosaurid theropods from the Lower Cretaceous Elrhaz Formation of Niger
}

\author{
Paul C. Sereno and Stephen L. Brusatte \\ Acta Palaeontologica Polonica 53 (1), 2008: 15-46 doi:http://dx.doi.org/10.4202/app.2008.0102
}

We report the discovery of basal abelisaurid and carcharodontosaurid theropods from the mid Cretaceous (Aptian-Albian, ca. $112 \mathrm{Ma}$ ) Elrhaz Formation of the Niger Republic. The abelisaurid, Kryptops palaios gen. et sp. nov., is represented by a single individual preserving the maxilla, pelvic girdle, vertebrae and ribs. Several features, including a maxilla textured externally by impressed vascular grooves and a narrow antorbital fossa, clearly place Kryptops palaios within Abelisauridae as its oldest known member. The carcharodontosaurid, Eocarcharia dinops gen. et sp. nov., is represented by several cranial bones and isolated teeth. Phylogenetic analysis places it as a basal carcharodontosaurid, similar to Acrocanthosaurus and less derived than Carcharodontosaurus and Giganotosaurus. The discovery of these taxa suggests that large body size and many of the derived cranial features of abelisaurids and carcharodontosaurids had already evolved by the mid Cretaceous. The presence of a close relative of the North American genus Acrocanthosaurus on Africa suggests that carcharodontosaurids had already achieved a trans-Tethyan distribution by the mid Cretaceous.

Key words: Theropod, abelisaurid, allosauroid, carcharodontosaurid, Kryptops, Eocarcharia, Cretaceous, Africa.

Paul C. Sereno [dinosaur@uchicago.edu], Department of Organismal Biology and Anatomy, University of Chicago, 1027 E. 57th Street, Chicago, Illinois, 60637, USA; Stephen L. Brusatte [brusatte@uchicago.edu], Department of Earth Sciences, University of Bristol, Wills Memorial Building, Queen's Road, Bristol BS8 1RJ, United Kingdom.

This is an open-access article distributed under the terms of the Creative Commons Attribution License (for details please see creativecommons.org), which permits unrestricted use, distribution, and reproduction in any medium, provided the original author and source are credited. 
FoF Full text $(2,077.9 \mathrm{kB})$ 